DOI: 10.1136/annrheumdis-2017-eular.3933

\section{SAT0653 QUANTITATIVE ASSESSMENT OF INTERSTITIAL LUNG DISEASE IN SJÖGREN SYNDROME: A PILOT STUDY}

P. Guisado Vasco ${ }^{1}$, M. Silva $^{2}$, N. Sverzellati ${ }^{2}$, I. Martín Garrido ${ }^{1}$, D. Santilli ${ }^{3}$, F. Mozzani ${ }^{3}$, O. Martin Segarra $^{1}$, J.M. Luque Pinilla ${ }^{1}$, A. Ariani ${ }^{4}$. ${ }^{1}$ Internal Medicine, Complejo Hospitalario Ruber Juan Bravo, Madrid, Spain; ${ }^{2}$ Radiology, University of Parma; ${ }^{3}$ Internal Medicine; ${ }^{4}$ Internal Medicine and Rheumatology unit, UAzienda Ospedaliero-Universitaria di Parmaniversity of Parma, Parma, Italy

Background: The interstitial lung disease (ILD) is the most frequent form of lung damage in Sjögren's syndrome (SjS). The diagnosis is still nowadays challenging: there are not specific test -autoantibodies, pulmonary function test- or symptoms. Chest CT is the gold standard. Semiquantitative (SQCT) assessment (such Taouli or Goh visual scores) could estimate ILD severity or burdened by relevant inter-rater variability. Quantitative chest CT (QCT) is a promising method to assess primary or secondary ILD, but there are not studies focused on SjS-ILD. Objectives: Herein we designed a study to evaluate if QCT indexes might assess differences in SjS-ILD.

Methods: We conducted a multicenter, cross-sectional, and retrospective study, identifying subjects affected of SjS (per modified American-European consensus criteria) and with a thorax CT ordered by local physician by any reason. We enrolled 52 cases, splitted in two cohorts according to the presence/absence of ILD on chest CT. A centralized SQCT assessment was carried out to calculate both Goh and Taouli scores. A DICOM-viewer open-source software (Horos) was used to analyse, blindly and anonymously, all CT images and to calculate QCT indexes (pulmonary $(\mathrm{p})$ and total $(\mathrm{t})$ kurtosis, standard deviation, skewness and mean lung attenuation). QCT indexes and SQCT scores were compared using the Spearman rank and Mann-Whitney tests. ROC curves were calculated to assess the sensibility and specificity of the QCT indexes to detect ILD.

Results: Median age was 70.5 (95\% Cl 65.47-73.0); 96.2\% were female. 9 cases have ILD, and 41 were not affected - two patients not assessable. The disease duration (till CT thorax) have a median of $21(95 \% \mathrm{Cl}-6-42.41)$ months. $42.3 \%$ have secondary SjS and $57.7 \%$ a primary SjS. All QCT scores, except tSDev, were statistically different in ILD comparing those without ILD; the most relevant were: pKurt 2.05 (IQR 0.389 - 3.78) vs 4.34 (IQR 2.74- 6.0), $\mathrm{p}=0.008$; pSdev (105.6 (IQR 101.4 - 122.95) vs 96.4 (IQR 92.38 to 104.94), $p=0.006$ ); pSkew (1.49 (IQR 0,49-1,94) vs 2.01 (IQR 1.59-2.38), p=0.011); tKurt 7.62 (IQR 3.35 - 9.31) vs 10.00 (IQR 8.60 - 11.42), $\mathrm{p}=0.012$ and tSkew 2.52 (1.63 $2.85)$ vs $3.04(2.81-3.29), p=0.005)$. ROC analysis showed that tSkew could best discriminate ILD and those without: $0.8(95 \% \mathrm{Cl} 0.66-0.9)$, cut off point $\leq 2.89$; sensitivity $88.89 \%(95 \% \mathrm{Cl} 51.8-99.7)$, specificity $65.85 \%$ (95\% Cl $49.4-79.9)$, $\mathrm{p}<0.001$. The correlation coefficients for QCT and Goh and Taouli's score ranges around \pm 0.4 .

Conclusions: QCT indexes identified some differences between SjS-ILD and those not. These parameters could be useful both to improve imaging diagnosis and also the decision to treat those cases. This innovative tool migh open up a potential research area to be developed in SjS. Moreover, the operator independence of QCT makes it a time-saving method extremely suitable for multi-centre trials. Our pilot study have some handicaps: small sample, low number of events, different reasons for CT performing, no smoking identification, and biological differences between primary and secondary SjS. However, it could be point out that QTC could be a useful tool to identify and quantify ILD in SjS, and further data are need to ascertain this hypothesis.

Disclosure of Interest: None declared

DOI: 10.1136/annrheumdis-2017-eular.6868

\section{SAT0654 THE RECALL SURVEY: DIFFERENCIES OF ULTRASOUND EXAMINATION BETWEEN EARLY VS LONG LASTING RHEUMATOID ARTHRITIS PATIENTS IN CLINICAL REMISSION}

P. Macchioni ${ }^{1}$, A. lagnocco ${ }^{2}$, M. Canzoni $^{3}$, C. Bonali ${ }^{4}$, O.M. Epis ${ }^{5}$,

E. Filippucci ${ }^{6}$, A. Delle Sedie ${ }^{7}$, S. Tropea ${ }^{8} .{ }^{1}$ Rheumatology, Arcispedale S Maria Nuova, Reggio Emilia; ${ }^{2} 2$ Academic Rheumatology Unit, Università degli Studi di Torino, Turin; ${ }^{3}$ Rheumatology, Local Health Unit (ASL)Rome 1, Rome;

${ }^{4}$ Rheumatology, Ospedale San Paolo, Bari; ${ }^{5}$ Rheumatology, Ospedale Niguarda Milano, Milan; ${ }^{6}$ Rheumatology, Università Politecnica delle Marche, Jesi (AN); ${ }^{7}$ Rheumatology, Università di Pisa, Pisa; ${ }^{8}$ Rheumatology, Busacca Hospital, Ragusa, Italy

Background: Ultrasound (US) examination is able to show subclinical synovitis and structural changes of joints in patients (pts) with RA in clinical remission (CR). Objectives: To investigate the differencies of US examination of small joints of hands, feet and wrists in pts with very early (VERA, <1y) vs early (ERA, <3y duration) vs long lasting RA (LRA, $>7$ y duration) in clinical remission (CR).

Methods: In 2015 an educational event (RECALL survey) focused on the added value of US in RA pts was held in 22 rheumatology centers in Italy. In every center, the local rheumatologists provided RA pts to be examined by US. Pts signed an informed consent and a brief history of them was collected by the local rheumatologists (previous and current therapy, DAS28, HAQ score). Bilateral US examinations of wrists, metacarpophalangeal (MCP) and metatarsophalangeal
(MTP) joints were performed by rheumatologists expert in US, to assess synovitis (joint effusion, synovial proliferation, and power Doppler (PD) signal), and bone erosions, using a Logiq E R7, General Electrics, with a 4.2-13 MHz linear probe. All US findings were scored using a 4 degree semiquantitative scoring system. Results: Eighty-eight pts were classified as VERA, 183 as ERA and 165 as LRA. Drug usage was not different among the three groups of pts. LRA patients were older $(p<0.001)$, had longer disease remission before US examination $(p<0.001)$ and had an higher prevalence of female sex $(p<0.01)$. Table 1 reports the differences of US examination between VRA vs ERA vs LRA patients. LRA patients had more erosions at MTP and wrists with no differences at MCP joints. Synovial hypertrophy and PD signal had the same degree and distribution in the two groups of patients. LRA patients had more MTP joints with effusion with lower degree as compared to patients with ERA and VERA (see table 1).

\begin{tabular}{|c|c|c|c|c|}
\hline & $\begin{array}{l}\text { VERA (A) } \\
88 \text { pts }\end{array}$ & $\begin{array}{l}\text { VERA+ERA } \\
183 \text { pts }\end{array}$ & $\begin{array}{l}\text { LRA }(C) \\
165 \mathrm{pts}\end{array}$ & $\mathrm{p}$ \\
\hline $\mathrm{M} / \mathrm{F}$ & $25 / 63$ & $58 / 125$ & $22 / 143$ & $\begin{array}{l}\text { A vs } C=0.002 \\
B \text { vs } C=<0.001\end{array}$ \\
\hline Number of pts aged $<50 y$ & $30 / 88$ & $58 / 183$ & $21 / 165$ & $\begin{array}{l}A \text { vs } C=<0.001 \\
B \text { vs } C=<0.001\end{array}$ \\
\hline$\%$ of pts with remission $<12$ months & 85 & 80 & 48 & $\begin{array}{l}A \text { vs } C=<0.001 \\
B \text { vs } C=<0.001\end{array}$ \\
\hline$\%$ of pts with $>1$ eroded joint & 39 & 54 & 62 & $\begin{array}{l}A \text { vs } C=<0.001 \\
B \text { vs } C=<0.001\end{array}$ \\
\hline $\begin{array}{l}\% \text { of pts with }>1 \text { joint with synovial } \\
\text { hypertrophy }\end{array}$ & 69 & 71 & 73 & $\begin{array}{l}A \text { vs } C=n s \\
B \text { vs } C=n s\end{array}$ \\
\hline$\%$ of pts with synovial hypertrophy $>1$ & 46 & 49 & 51 & $\begin{array}{l}A \text { vs } C=n s \\
B \text { vs } C=n s\end{array}$ \\
\hline$\%$ of pts with $>1$ joint positive for PD signal & 48 & 50 & 45 & $\begin{array}{l}A \text { vs } C=n s \\
B \text { vs } C=n s\end{array}$ \\
\hline$\%$ of joints with PD signal $>1$ & 32 & 34 & 35 & $\begin{array}{l}A \text { vs } C=n s \\
B \text { vs } C=n s\end{array}$ \\
\hline$\%$ of pts with effusion at MTP joints & 45 & 49 & 59 & $\begin{array}{l}A \text { vs } C=0.034 \\
B \text { vs } C=0.044\end{array}$ \\
\hline$\%$ of pts with MTP joints effusion $>1$ & 25 & 21 & 12 & $\begin{array}{l}A \text { vs } C=0.010 \\
B \text { vs } C=0.023\end{array}$ \\
\hline
\end{tabular}

Conclusions: At US examination VERA, ERA and LRA pts in CR have the same degree of synovial hyperplasia and PD signal despite the differences of duration of CR. Higher degree of erosive disease (at wrist and MTP joints), lower degree of MTP effusion and higher prevalence of joint effusion at MTP joints were present among LRA vs ERA and VERA pts.

Disclosure of Interest: None declared

DOI: 10.1136/annrheumdis-2017-eular.5047

\section{SAT0655 EARLY ENDOTHELIAL DAMAGE IN PATIENTS WITH RAYNAUD'S PHENOMENON}

R. Gualtierotti ${ }^{1,2}$, F. Ingegnoli ${ }^{2,3}$, T. Schioppo $^{2}$, S. Griffini ${ }^{4,5}$, E. Grovetti ${ }^{4,5}$, M.O. Borghi ${ }^{6}$, M. Cugno ${ }^{4,5}$, P.L. Meroni ${ }^{1,2,3,6}$. ' ${ }^{1}$ Lupus Clinic; ${ }^{2}$ Division of Rheumatology, ASST G. Pini; ${ }^{3}$ Department of Clinical Sciences and Community Health; ${ }^{4}$ Dipartimento di Fisiopatologia Medico-Chirurgica e dei Trapianti, University of Milan; ${ }^{5}$ Medicina Interna, Ospedale Maggiore Policlinico, Fondazione IRCCS Ca' Granda; ${ }^{6}$ IRCCS Istituto Auxologico Italiano, Milan, Italy

Background: Raynaud's phenomenon (RP) can be the first manifestation of systemic sclerosis (SSc) or other connective tissue diseases (CTDs), often preceding an overt disease by years. It is not known if markers of endothelial damage are detectable in those RP patients who subsequently develop a CTD.

Objectives: We studied RP patients at their first evaluation to correlate the levels of endothelial markers with the subsequent development of an overt disease 36 months later.

Methods: Eighty-two patients with RP at their first evaluation were recruited. We measured plasma levels of tissue-type plasminogen activator (t-PA) and von Willebrand factor (vWF), two markers of endothelial damage, and interleukin-6 (IL-6), a pro-inflammatory cytokine. Thirty healthy subjects served as healthy controls.

Results: At baseline, 67 patients showed capillaroscopic normal pattern and 15 patients, of which 11 were very early SSc, had scleroderma pattern. Plasma levels of t-PA, vWF and IL-6 were higher in patients with capillaroscopic normal pattern $(p=0.0001)$ than in normal controls and even much higher in patients with scleroderma pattern $(p=0.0001)$. In patients with capillaroscopic normal pattern and RP of recent onset ( $<18$ months), vWF plasma levels were higher when autoantibodies were present $(p=0.020)$. After 36 months, among 48 RP patients with capillaroscopic normal pattern who remained in follow-up, 24 were diagnosed as primary and 24 as secondary RP. In secondary RP, basal levels of t-PA, IL- 6 and particularly vWF were higher than in primary RP $(p=0.005, p=0.004$, $p=0.0001$ respectively) and normal controls ( $p=0.0001$ for all).

Conclusions: Our findings indicate that markers of endothelial damage are elevated in RP patients who subsequently develop SSc or other CTDs, even in the absence of capillaroscopic abnormalities.

Disclosure of Interest: None declared

DOI: 10.1136/annrheumdis-2017-eular.5416 